\title{
The Tacking by Disjunction Paradox: Bayesianism versus
}

\section{Hypothetico-deductivism}

\author{
Luca Moretti \\ Center for Junior Research Fellows \\ University of Konstanz \\ Luca.Moretti@uni-konstanz.de
}

\begin{abstract}
Hypothetico-deductivists have struggled to develop qualitative confirmation theories not raising the so-called tacking by disjunction paradox. In this paper, I analyze the difficulties yielded by the paradox and argue that the hypothetico-deductivist solutions given by Gemes (1998) and Kuipers (2000) are questionable because they do not fit such analysis. I then show that the paradox yields no difficulty for the Bayesian who appeals to the Total Evidence Condition. I finally argue that the same strategy is unavailable to the hypothetico-deductivist.
\end{abstract}

\section{Introduction: Hypothetico-deductivism and the tacking by disjunction paradox}

In this paper, I focus on the so-called tacking by disjunction paradox. I show that, though the hypothetico-deductivist attempts to resolve this paradox made by Gemes (1998) and Kuipers (2000) are questionable, the Bayesian who endorses the Total Evidence Condition can settle the paradox without any apparent difficulty.

A crude formulation of Hypothetico-deductivism says that:

(HD) For any statements $H$ and $E, E$ confirms $H$ if $H$ and $E$ are contingent, $E$ is a logical consequence of $H$, and $E$ is verified. (Cf. Grimes 1990: 515).

Some philosophers prefer to define Hypothetico-deductivism by appealing to the notion of confirmation relative to (or given) background knowledge. The adduced reason is, usually, that in 
actual science background knowledge does play a role in deduction and confirmation. A simple formulation of relative Hypothetico-deductivism is the following:

(RHD) For any statements $H, E$ and $b, E$ confirms $H$ relative to $b$ if $H \& b$ and $E$ are contingent, $E$ is a logical consequence of $H \& b$ but not of $b$ alone, and $E$ is verified.

I will hereafter focus on (RHD) rather than on (HD), as the former principle appears to reflect scientific practice more accurately.

A difficulty for Hypothetico-deductivism is the tacking by disjunction paradox. If Hypothetico-deductivism is defined by (RHD), the tacking by disjunction paradox hinges on the following counterintuitive confirmation rule:

(TD) For any statements $H, E$ and $b$, if $H \& b$ is contingent and entails $E$, for any arbitrary statement $E^{*}$, if $E \vee E^{*}$ is contingent and is not entailed by $b$ alone, whenever $E \vee E^{*}$ is verified, $E \vee E^{*}$ confirms $H$ relative to $b$.

(TD) follows from (RHD) by introduction of the disjunction (if $X$ entails $Y$, then, for whatever $Z, X$ entails $Y \vee Z$ ). ${ }^{1}$ Roughly, the trouble with (TD) is that $E^{*}$ may appear irrelevant to $H^{\prime}$ 's confirmation given $b$ (in the intuitive sense that if one verifies $E^{*}$, this does not affect one's confidence in $H$ given $b$ ) or $E^{*}$ may even be inconsistent with $H \& b$. One can thus feel reluctant to admit that the verification of $E \vee E^{*}$ does confirm $H$ given $b$.

Let us call any statement $E \vee E^{*}$ such that $H \& b$ entails $E, E$ appears positively relevant to $H$ given $b$ (in the intuitive sense that one's verification of $E^{*}$ increases one's confidence in $H$ given $b$ ), and $E^{*}$ appears irrelevant to $H$ given $b$ or is inconsistent with $H \& b$, a tacked disjunction for $H$ given $b$, or simply a tacked disjunction. Consider for example the case in which a hypothesis $H$ has 
the form of the generalization $(x)(F x), E$ is equivalent to $F a$, and $E^{*}$ is equivalent to $R a$ or to $\neg F c$. Notice that $\neg F c$ is logically inconsistent with $(x)(F x) \& b$, and $R a$ seems irrelevant to $(x)(F x)$ given $b$ if $b$ is tautologous. The first impression may be that the verification of the tacked disjunctions $F a$ $\vee R a$ and $F a \vee \neg F c$ should not confirm the hypothesis $(x)(F x)$ given $b$. This impression can be strengthened by other considerations. For example, it can be argued that if one allows tacked disjunctions to confirm hypotheses, for any statement $H$ however idiotic, as long as $H$ is contingent and there is some other verified statement $E^{*}$ such that $H \vee E^{*}$ is contingent, one has a confirmation of $H$. Thus the foolish claim 'The moon is made of Green cheese' is confirmed by the verification of 'The moon is made of Green cheese or London is in the UK', which is unacceptable.

In Section 2, I show that the tacking by disjunction problem is much less univocal than one might expect: the verification of a tacked disjunction $E \vee E^{*}$ appears in certain cases not to confirm $H$ given $b$ but, in other cases, to confirm it. Any adequate solution of the tacking by disjunction paradox should distinguish the cases in which $E \vee E^{*}$ confirms $H$ from those in which it does not. In Sections 3 and 4, I examine the two most recent hypothetico-deductivist attempts to resolve this paradox, given in Gemes (1998) and in Kuipers (2000). I argue that both solutions are inadequate because they do not distinguish the cases in which $E \vee E^{*}$ confirms $H$ from those in which it does not. In Section 5, I show that the Bayesian who accepts Total Evidence Condition as a constraint for the application of the rule of incremental confirmation can settle the paradox without falling afoul of similar difficulties. Finally, in the conclusion of the paper, I argue that the application of the Total Evidence Condition is hampered by serious difficulties when hypothetico-deductivist analysis of confirmation is presupposed.

\section{An analysis of the tacking by disjunction paradox}

Consider three statements $H, E$ and $b$ such that $H \& b$ is contingent, $H \& b$, but not $b$ alone, entails $E$, and $E$ appears positively relevant for $H$ given $b$. Furthermore, let $E^{*}$ be a statement that appears 
irrelevant for $H$ given $b$ or, alternatively, that is inconsistent with $H \& b$, and such that $E \vee E^{*}$ is contingent and is not entailed by $b$. In accordance with (TD), $E \vee E^{*}$, whenever verified, confirms $H$ given $b$. This can happen in four cases: (I) if only $E$ but not $E^{*}$ is verified, (II) if only $E^{*}$ but not $E$ is verified, (III) if both $E$ and $E^{*}$ are verified and, finally, (IV) if neither $E$ nor $E^{*}$ is verified, though $E \vee E^{*}$ is (I provide two examples of this below). Each of these cases can be divided into two sub-cases depending on (-irr) if $E^{*}$ is irrelevant to $H$ given $b$ or (-inc) if $E^{*}$ is inconsistent with $H \& b$. Notice that other possible sub-cases could be individuated. For example, $E^{*}$ may appear negatively relevant for $H$ given $b$, in the sense that one's verification of $E^{*}$ lessens one's confidence in $H$ given $b$ without necessarily nullifying it (as when $E^{*}$ is inconsistent with $H \& b$ ). For the sake of simplicity, I will however focus only on the two extreme sub-cases (-irr) and (-inc); this fully suffices for the purposes of this paper.

Let us now try to determine whether, in the mentioned cases and sub-cases, one's verification of $E \vee E^{*}$ confirms or does not confirm $H$ given $b$, in the intuitive and pre-theoretical sense of increasing one's confidence in $H$ given $b .^{2}$

Case (I): only $E$ but not $E^{*}$ is verified.

If one verifies $E \vee E^{*}$ by verifying just $E$, whether $E^{*}$ is irrelevant to $H$ given $b$ or inconsistent with $H \& b$, it is straightforward that one's confidence in $H$ given $b$ increases. If $E^{*}$ is inconsistent with $H \& b$, this does not undermine one's confidence in $H$ given $b$, as $E^{*}$ is not verified. In conclusion, in both (I-irr) and (I-inc), the verification of $E \vee E^{*}$ does result in the confirmation of $H$ given $b$.

Case (II): only $E^{*}$ but not $E$ is verified.

Let us first focus on (II-irr). If one verifies $E \vee E^{*}$ by verifying just $E^{*}$, and $E^{*}$ is irrelevant to $H$ given $b$, one's confidence in $H$ given $b$ does not increase. Consider for example the situation in which $H$ is the General Relativity theory or one of its axioms, $E$ is a consequence of $H$ and my background knowledge $b$ that is not verifiable on the grounds of present technology, and $E^{*}$ is the 
statement 'In the left pocket of my jacket there is a penny'. Suppose I have actually verified that I have a penny in the left pocket of my jacket: $E \vee E^{*}$ is thus verified. Yet this does not increase my confidence in the axioms of the General Relativity theory! In this case, the verification $E \vee E^{*}$ does not confirm $H$ given $b$.

Let us turn to (II-inc). If one verifies $E \vee E^{*}$ by verifying just $E^{*}$, and $E^{*}$ is inconsistent with $H$ $\& b$, one's confidence in $H$ will not increase, as one has to conclude that $H$ is false (assuming that $b$ is true). Consequently, the verification of $E \vee E^{*}$ cannot confirm $H$ given $b$. To summarize, in both (II-irr) and (II-inc), the verification $E \vee E^{*}$ does not result in the confirmation of $H$ given $b$.

Case (III): both $E^{*}$ and $E$ are verified.

Let us first consider (III-irr). If one verifies $E \vee E^{*}$ by verifying both $E$ and $E^{*}$, and $E^{*}$ is irrelevant to $H$ given $b$, it is intuitive that one's confidence in $H$ given $b$ increases in many cases. For example, this plausibly happens if I verify both disjuncts $E$ and $E^{*}$ in the example about the General Relativity theory given in case (II-irr). Yet, to see that this sometimes may not happen, think of the following possible situation: $E^{*}$ is equivalent to $E \& F$, where the verification of $F$ annuls the boosting effect of the verification of $E$ on my confidence in $H$ given $b$. Clearly, if I verify both $E$ and $E^{*}$, my confidence in $H$ does not increase.

Let us now consider (III-inc). If one verifies $E \vee E^{*}$ by verifying both $E$ and $E^{*}$, and $E^{*}$ is inconsistent with $H \& b$, one's confidence in $H$ given $b$ is nullified. For one has to conclude that $H$ is false (assuming that $b$ is true). To summarize, in (III-irr), the verification $E \vee E^{*}$ confirms $H$ given $b$ in many cases, and in (III-inc) the verification of $E \vee E^{*}$ does not confirm $H$ given $b$.

Case (IV): neither $E$ nor $E^{*}$ is verified, though $E \vee E^{*}$ is.

Let us first focus on (IV-irr). $E^{*}$ is irrelevant to $H$ given $b$. In addition, neither $E$ nor $E^{*}$ is verified though $E \vee E^{*}$ is. When these conditions are satisfied, there are many cases in which the verification of $E \vee E^{*}$ results in the confirmation of $H$ given $b$. Consider the following example: I have to evaluate the hypothesis $H$ that $\mathrm{Mr} X$ 's is a Russian academic, which entails (in normal 
conditions) $)^{3}$ that there is a paper in Russian that $\mathrm{Mr} X$ can read. Suppose, for instance, that I am in a room of a library where, on a desk, there is a pile of books in which there is only a red book. My background knowledge $b$ includes the following relevant information: the room contains just two red books, of which one is in Russian and the other in French, and any other book in the room is in French. Suppose now that $\operatorname{Mr} X$ approaches the desk, he looks at the whole pile of books, but picks up just the red book and starts reading it. $E$ is the positively relevant statement, entailed by $H \& b$, that there is a paper in Russian that $\operatorname{Mr} X$ can read and $E^{*}$ is the irrelevant statement that there is a paper in French that $\mathrm{Mr} X$ can read. All my evidence indicates that the contingent disjunction $E \vee$ $E^{*}$ is true, though I do not know which one of the disjuncts is true. It is quite intuitive that, in such a situation, the verification of $E \vee E^{*}$ is positively relevant for $H$ 's confirmation given $b$. For, now, I am a little bit more confident than before that $\mathrm{Mr} X$ is a Russian academic. Similar examples can easily be invented.

Let us turn to (IV-inc). $E^{*}$ is inconsistent with $H \& b$; moreover, neither $E$ nor $E^{*}$ is verified, though $E \vee E^{*}$ as a whole is. When these conditions are satisfied, again, there are many cases in which it is intuitive that $E \vee E^{*}$ confirms $H$ given $b$. Consider again the example about $\operatorname{Mr} X$. My background knowledge $b$ still includes the relevant information that the room in the library contains just two red books, of which one is in Russian and the other in French, and that all other books are in French. The only change in $b$ is that, now, I also know that - for some reason - whoever has access to the library and reads French is not a Russian academic. This renders $E^{*}$ inconsistent with $H \& b$ (though $E \vee E^{*}$ remains contingent). Suppose again that $\operatorname{Mr} X$ picks up just the red book from the pile and starts reading it. In these slightly changed circumstances, the verification of $E \vee$ $E^{*}$ still appears positively relevant for $H$ 's confirmation given $b$. For, as I verify $E \vee E^{*}$ without verifying either disjunct, I feel a little bit more confident than before that $\operatorname{Mr} X$ is a Russian academic. Similar examples can easily be made up. In conclusion, in both (IV-irr) and (IV-inc), the verification $E \vee E^{*}$ results in the confirmation of $H$ given $b$ in many cases. 
The following table summarizes the results of my analysis.

(irr) $\underline{E^{*} \text { is irrelevant to } H \text { given } b}$ (inc) $E^{*}$ is inconsistent with $H \& b$

(I) $\underline{E \text { is verified but not } E^{*}} \quad E \vee E^{*}$ confirms $H$ given $b \quad E \vee E^{*}$ confirms $H$ given $b$

(II) $\underline{E^{*} \text { is verified but not } E} \quad E \vee E^{*}$ does not confirm $H \quad E \vee E^{*}$ does not confirm $H$ given $b \quad$ given $b$

(III) $\underline{\text { Both } E^{*} \text { and } E \text { are }}$ verified

$E \vee E^{*}$ confirms $H$ given $b$ in many cases

$E \vee E^{*}$ confirms $H$ given $b$ in many cases
$E \vee E^{*}$ does not confirm $H$ given $b$

$E \vee E^{*}$ confirms $H$ given $b$ in many cases
(IV) $\underline{E \vee E^{*} \text { is verified but }}$ neither $E$ nor $E^{*}$ is

\footnotetext{
.
}

Notice finally that if one allows tacked disjunctions to confirm hypotheses in accordance with the above table, one need not conclude that any contingent but foolish statement is confirmable. For such a counterintuitive conclusion follows only if one allows tacked disjunctions to confirm hypotheses indiscriminately.

\section{Gemes' hypothetico-deductivist solution}

Philosophers have struggled to provide formulations of Hypothetico-deductivism that either do not entail rules like (TD) or that try to limit the paradoxical consequences of them. In this section, I will focus on the formulation of Hypothetico-deductivism given by Gemes (1998), which does not entail (TD). In the next section, I will examine an alternative version of Hypothetico-deductivism given by Kuipers (2000), which, while entailing (TD), tries to limit its paradoxical consequences.

Although Gemes believes that Hypothetico-deductivism provides no adequate account of confirmation (see below), he aims to give the best formulation of this methodology. To accomplish this task, Gemes (1994), (1997) and, in particular, (1998) has persuasively refuted a number of 
different formulations of Hypothetico-deductivism, particularly those given in Schurz (1991) and (1994) and in Grimes (1990), which also put forward solutions for the tacking by disjunction paradox.

Gemes formulates Hypothetico-deductivism following Hempel's intuition that a statement confirms a hypothesis if the statement is part of the hypothesis' content, i.e. part of what it asserts about the world. ${ }^{4}$ To articulate this intuition, Gemes first provides a precise account of content. On his view, given two statements $\alpha$ and $\beta$,

$\alpha$ is part of the content of $\beta$ iff $\alpha$ and $\beta$ are contingent, $\alpha$ is a consequence of $\beta$, and every relevant model of $\alpha$ can be extended to a relevant model of $\beta$. (Gemes 1998: 9).

Gemes has elucidated the notion of a relevant model as follows:

A relevant model for a wff $\alpha$ is an assignment of truth-values to each and only those atomic wffs relevant to $\alpha$ such that every full extension of that assignment is a model of $\alpha$. For instance, the only atomic wffs relevant to ' $F a \& F b$ ', are ' $F a$ ' and ' $F b$ ' and the only relevant model for ' $F a \& F b$ ' is that assignment which assigns $T$ [i.e. the truth] to both ' $F a$ ' and ' $F b$ ' and makes no other assignments. The only atomic wffs relevant to ' $F a \vee \neg F b$ ' are ' $F a$ ' and ' $F b$ '. However that relevant model of ' $F a \vee \neg F b$ ' that assigns ' $F a$ ' the value $T$ and ' $F b$ ' the value $F$ and makes no other assignment cannot be extended to a relevant model for ' $F a \& F b$ '. So ' $F a \vee \neg F b$ ' is not part of the content of ' $F a \& F b$ '. On the other hand, ' $F a$ ' is a content part of ' $F a \& F b$ ' since ' $F a$ ' has only one relevant model, namely that model that assigns $T$ to ' $\mathrm{Fa}$ ' and makes no other assignment, and that model can clearly be extended to a relevant model for ' $F a \& F b$ '. (Ibid.). 
Gemes' notions of a relevant model and content also apply to wffs containing quantifiers, where the quantifiers are treated substitutionally. (Cf. ibid.). For instance, the atomic wffs relevant to $(x)(F x \& G x)$ are $F a, F b, F c, \ldots$ and $G a, G b, G c, \ldots$ and the only relevant model for $(x)(F x \& G x)$ is the model that assigns $T$ to both the atomic wffs $F a, F b, F c, \ldots$ and the atomic wffs $G a, G b, G c, \ldots$ and makes no other assignments. $F a$ is part of the content of $(x)(F x \& G x)$, for the only relevant model for $F a$ (i.e., the model that assigns $T$ to $F a$ and that makes no other assignment) can be extended to a relevant model of $(x)(F x \& G x)$. Oppositely, $(\exists x) F x$ is not part of the content of $(\mathrm{x})(F x$ $\& G x)$ because the relevant model of $(\exists x) F x$ that assigns $T$ to $F a$ and $F$ to $F b, F c, F d$, ... cannot be extended to a relevant model for $(x)(F x \& G x)$.

After this, Gemes gives the following definition of a natural axiomatization of a theory $T$, where a theory is any set of wffs closed under the logical consequence relation:

$T^{*}$ is a natural axiomatization of $T$ iff (i) $T^{*}$ is a finite set of wffs such that $T^{*}$ is logically equivalent to $T$, (ii) every member of $T^{*}$ is a content part of $T^{*}$ and (iii) no content part of any member of $T^{*}$ is entailed by the set of the remaining members of $T^{*}$. (Ibid.).

For instance, $\{F a, F b\}$ is a natural axiomatization of the theory consisting of the deductive closure of $\{F a, F b\}$. Quite the opposite, $\{F a, F a \rightarrow F b\}$ is not a natural axiomatization of the deductive closure of $\{F a, F b\}$, though $\{F a, F a \rightarrow F b\}$ is logically equivalent to $\{F a, F b\}$. For $F a$ $\rightarrow F b$ is a member of $\{F a, F a \rightarrow F b\}$ but it is not a content part of it because the relevant model of $F a \rightarrow F b$ that assigns $F$ to $F a$ and $T$ to $F b$ cannot be extended to a relevant model of $\{F a, F a \rightarrow$ Fb\}. (Cf. ibid.: 9-10).

On the grounds of these notions of content and natural axiomatization, Gemes provides the following sophisticated version of relative Hypothetico-deductivism, which is meant to sort out different confirmation paradoxes including the tacking by disjunction one: 
(RHD1) Where $N(T)$ is a natural axiomatization of theory $T$ and $H$ is an axiom of $N(T)$, evidence $E$ hypothetico-deductively confirms axiom $H$ of theory $T$ relative to background evidence $b$ iff, $E$ and (non-tautologous) $b$ are content parts of $T \& b$, and there is no natural axiomatization, $N(T)^{*}$ of $T$ such that for some subset $s$ of the axioms of $N(T)^{*}, E$ is a content part of $s \& b$ and $H$ is not a content part of $s \& b$. (Ibid.: 10).

The intuition standing behind (RHD1) is that if $E$ is a content part $T \& b, E$ confirms only the natural axioms of $T$ indispensable for the deduction of $E$. Referring confirmation not to $T$ but to an axiom $H$ of $T$ is useful, on Gemes' view, to settle the so-called tacking by conjunction paradox. ${ }^{5}$

Finally, Gemes emphasizes that:

[(RHD1)] solves the problem of the tacking by disjunction, since, where $E$ is a content part of $T$, it does not follow that for arbitrary $E^{*}, E \vee E^{*}$ is part of the content of $T \& b$. For instance, while ' $F a$ ' is part of the content of ' $(x) F x$ ', ' $F a \vee G b$ ' is not part of the content of ' $(x) F x$ '. (Ibid.).

Gemes' solution is reducible to the claim that (RHD1) in most cases does not allow a tacked disjunction for a given hypothesis $H$, which is a natural axiom of a theory, to confirm that hypothesis. This can easily be shown. A statement $E \vee E^{*}$ is a tacked disjunction for a natural axiom $H$ of a theory $T$ given $b$ if and only if $H \& b$ entails $E, E$ appears positively relevant to $H$ given $b$, and $E^{*}$ appears irrelevant to $H$ given $b$ or is inconsistent with $H \& b$. If $E^{*}$ is inconsistent with $H \& b, E^{*}$ is also inconsistent with $T \& b$. Consequently, $E \vee E^{*}$ cannot be part of the content of $T \& b$, as the relevant models of $E \vee E^{*}$ that make $E^{*}$ true cannot be extended to relevant models 
of $T \& b$. This entails that $E \vee E^{*}$ cannot confirm $H$ given $b$ on (RHD1). It is also intuitive that when $E^{*}$ appears irrelevant to $H$ given $b$, in most cases, $E \vee E^{*}$ will not be part of the content of $H$ $\& b$. It follows from (RHD1) that if $H \& b$ entails $X$ and $X$ is not part of the content of $H \& b, X$ does not confirm $H$ given $b .^{6}$ Thus, when $E^{*}$ appears irrelevant to $H$ given $b$, in most cases, $E \vee E^{*}$ will not confirm $H$ given $b$.

Interestingly, if $E^{*}$ is irrelevant for a natural axiom $H$ of a theory $T$ given $b$, the tacked disjunction $E \vee E^{*}$ for $H$ given $b$ will sometimes turn out to confirm $H$ given $b$ on (RHD1). Suppose that $E$ confirms $H$ given $b$ on (RHD1). (If Gemes' hypothetico-deductive account of confirmation is correct, if $H \& b$ entails $E$ and $E$ appears positively relevant to $H$ given $b, E$ should indeed confirm $H$ given $b$ on (RHD1)). Assume also that $E^{*}$ is irrelevant to $H$ given $b$ because $E^{*}$ is equivalent to $E$ $\& F$, where the verification of $F$ annuls the boosting effect of the verification of $E$ on one's confidence in $H$ given $b$. Since $E$ and $E \vee E^{*}$ prove logically equivalent and, by assumption, $E$ confirms $H$ given $b, E \vee E^{*}$ will also confirm $H$ given $b$.

Notice (RHD1) does not require a statement $E$ to be verified to confirm $H$ given $b$, for Gemes conceives of confirmation in terms of purely logical relationships among statements. (RHD1) can nevertheless be used to ascertain whether $H$ is confirmed by evidence that one actually has if $E$ is taken to represent such actual evidence. The results of the analysis of the tacking by disjunction paradox can be (trivially) re-formulated in terms of actual evidence rather than in terms of verified statements. For instance, case (I) can be re-described as the one in which $E \vee E^{*}$ is evidence that one actually has because only $E$, but not $E^{*}$, is evidence that one actually has, and so on. The trouble with Gemes' solution is, then, that it does too much. For it allows a tacked disjunction for a given hypothesis to confirm that hypothesis in almost no case, while there are many cases in which tacked disjunctions that represent actual evidence should confirm hypotheses. In particular, though (RHD1) rules out the intuitively incorrect cases of confirmation (II-inc) and (III-inc), it also disallows the intuitively correct cases of confirmation (I-inc), the correct ones included in (IV-inc) 
and, very probably, many of the intuitively correct cases of confirmation (I-irr) and many of the genuine ones included in (III-irr) and (IV-irr). There are further problems: I have shown above that, in a specific case, when $E^{*}$ is irrelevant to $H$ given $b$, (RHD1) permits $E \vee E^{*}$ to confirm $H$ given $b$. Consequently, (RHD1) will allow for confirmation cases in situations (II-irr) and (III-irr), which should instead be forbidden.

Notice that (RHD1) gives conditions for which evidence hypothetico-deductively confirms a hypothesis. This does not undermine my criticism. Hypothetico-deductive method is meant to account for all cases of deductive confirmation, and the intuitively correct cases of confirmation that (RHD1) disallows are just of this type, as the disjunction $E \vee E^{*}$ is always logically deducible from $H \& b$. It could however be argued that the fact that (RHD1) prohibits correct confirmation cases in situations (I-irr), (I-inc) and (III-irr) yields in practice no difficulty, as all these cases are redundant. For every time $E \vee E^{*}$ confirms $H$ given $b$ in such situations, $E$ alone confirms $H$ given $b$. This makes (I-irr), (I-inc) and (III-irr) dispensable if all one wants to know is whether actual evidence confirms $H$ given $b$. Although this is probably correct, it does not resolve all difficulties affecting (RHD1). For Gemes' Hypothetico-deductivism also entails that (IV-inc) is not a case of confirmation, which appears false in great many situations. In addition, (RHD1) will plausibly debar many genuine cases of confirmation included in (IV-irr), and it will allow counterintuitive confirmation cases in situations (II-irr) and (III-irr).

In conclusion, Gemes' solution of the tacking by disjunction paradox is not satisfactory. If (RHD1) is actually the best formulation of Hypothetico-deductivism, this conclusion goes with Gemes' very conviction that Hypothetico-deductivism is unable to provide an adequate account of confirmation. This does not make my analysis superfluous or uninteresting, as I have provided different reasons to strengthen the same conviction. Gemes' complaint is that Hypotheticodeductivism 'is not sufficiently inductivist' (ibid.: 11) because it cannot explain cases in which statements are intuitively confirmed by evidence that they do not entail. ${ }^{7}$ What I have shown is that 
Hypothetico-deductivism is defective (at least, in Gemes' formulation) because it cannot account for cases in which statements are intuitively confirmed or not confirmed by evidence that they do entail. $^{8}$

\section{Kuipers' hypothetico-deductivist solution}

Kuipers (2000)'s solution differs from Gemes' in that instead of re-constructing a version of Hypothetico-deductivism that no longer entails problematic rules like (TD), rather Kuipers reformulates Hypothetico-deductivism in a way that limits (TD)'s paradoxical consequences. Besides, Kuipers' Hypothetico-deductivism does not attempt to play out Hempel's intuition about the connection between confirmation and content, as Gemes' does. In what follows, I will present a simplified version of Kuipers' complicated formulation of Hypothetico-deductivism that nevertheless retains the essence of the solution of the tacking by disjunction paradox stemming from the original version.

Kuipers emphasizes that, though, 'Traditionally, [Hypothetico-deductivism as] a qualitative theory of deductive confirmation is conceived as merely a classificatory theory' (ibid.: 21), he intends to 'conceive it from the outset as a combination of classificatory and comparative principles' (ibid.). Such a broadened view would make available solutions for a number of different problems affecting theory confirmation.

According to Kuipers:

Classificatory notions of evaluation of a hypothesis $H$ by evidence $E$ are generated by considering the four possible, mutually exclusive [...], deductive relations [relative to $H, E$ and their logical negations] in a systematic order, assuming that $H[\ldots \text { and } \neg E \text { are both }]^{9}$ consistent and $E$ is true. (Ibid.: 19). 
In particular, the classificatory notion of (deductive) confirmation is generated when the deductive relation exists between $H$ and $E$ - precisely, $E$ confirms $H$ when $H$ entails $E$. (Cf. ibid.: 21-22). ${ }^{10}$ Confirmation should moreover be thought of as 'a three place relation between evidence, hypothesis and background beliefs'. (Ibid.: 23).

In conclusion, Kuipers appears to think of (RHD), introduced in the beginning of this paper, as the central classificatory principle of relative Hypothetico-deductivism. That is:

(RHD) For any statements $H, E$ and $b, E$ confirms $H$ relative to $b$ if $H \& b$ and $E$ are contingent, $E$ is a logical consequence of $H \& b$ but not of $b$ alone, and $E$ is verified.

As we know, (RHD) entails the problematic rule (TD), which the paradox of the tacking by disjunction rests on.

Kuipers supplements (RHD) with several principles to make comparative evaluations among theories, and that 'are roughly in agreement with scientific common sense' (ibid.: 24). These principles lead to the following, derived principle:

(P.1) If $E$ and $F$ confirm $H$ relative to $b$ then $E$ confirms $H$ relative to $b$ more than $F$ iff $E$ is less plausible than $F$ relative to $b$. (Cf. ibid.).

Here, when we say that ' $E$ is less plausible than $F$ relative to $b$ ', we mean that before $E$ and $F$ are verified, it is reasonable for an agent to be more confident in $F$ than in $E$ relative $b$.

How can this machinery provide any solution to the tacking by disjunction paradox? Kuipers points out that, under the intuitive assumption that logically weaker evidence is more plausible than stronger evidence, (P.1) entails that, for non-equivalent $E$ and $F$ : 
(S.1) If $H \& b$ entails $E$ and $E$ entails $F$, then $E$ confirms $H$ relative to $b$ more than $F$. (Cf. ibid.: 25).

(S.1) would finally allow for a solution of the problem at stake, as it would limit the problematic consequences of (TD). As Kuipers explain:

If $E$ confirms $H$ [relative to $b$ ] then

$-E \vee E^{*}$ confirms $H$ [relative to $\left.b\right]$, for any $E^{*}$ consistent with $E$

- though (much) less (due to S.1)

- but $E^{*}$ does not necessarily confirm $H$ [relative to $\left.b\right]$,

- hence, the confirmation remains perfectly localizable. (Ibid.: 26).

Unfortunately, Kuipers' ingenious solution does not comply with intuitive judgments about $H$ 's confirmation in the distinct cases in which the disjunction $E \vee E^{*}$ is verified. A difference between Gemes' solution and Kuipers' is that, while the former does too much, the latter does too little. As it allows a (contingent) tacked disjunction for a given (contingent) hypothesis to confirm the hypothesis in all cases.

Suppose that $E \vee E^{*}$ is a tacked disjunction for $H$ given $b$. Kuipers' claim that $E \vee E^{*}$ confirms $H$ given $b$ (less than $E$ ) accounts for the situations (I-irr) and (I-inc) and the cases of confirmation included in (III-irr), (IV-irr) and (IV-inc). Yet Kuipers' claim also entails that (II-irr), (II-inc) and (III-inc) are cases of confirmation, when it is straightforward that in these situations $E \vee E^{*}$ cannot confirm $H$ given $b$. In (II-irr), (II-inc) and (III-inc), Kuipers' notion of confirmation clashes with quite definite intuitions about confirmation. Kuipers' observation that ' $E *$ does not necessarily confirm $H$ relative to $b$ - hence the confirmation remains perfectly localizable' does not make up for this mismatch. For the problem with (II-irr), (II-inc) and (III-inc) is that $E \vee E^{*}-$ and not $E^{*}-$ 
should not confirm $H$ given $b$. Notice finally that Kuipers' framework entails the counterintuitive result that any contingent but foolish statement - like 'the moon is made of Green Cheese' - is confirmable. In conclusion, Kuipers' elaborate formulation of Hypothetico-deductivism offers a solution of the tacking by disjunction paradox that may appear even more defective than that proposed by Gemes. ${ }^{11}$

\section{The Bayesian solution: the appeal to the Total Evidence Condition}

Although the hypothetico-deductivists are far from providing one satisfactory solution of the tacking by disjunction paradox, the Bayesian methodologist has at her hand a simple solution that fully matches our insight into confirmation. Consider a standard language of propositional logic and a finitely additive Kolmogorov probability function $\operatorname{Pr}$ on the language. $\operatorname{Pr}$ is interpreted subjectively, in the sense that if $X$ is a statement of the language, $\operatorname{Pr}(X)$ gives the degree of confidence of a rational agent in $X^{\prime}$ s truth. The probability of $X$ conditional on another statement $Y$ of the language is defined as $\operatorname{Pr}(X \mid Y)=\operatorname{Pr}(X \& Y) / \operatorname{Pr}(Y)$, provided that $\operatorname{Pr}(Y)>0 . \operatorname{Pr}(X \mid Y)$ measures a rational agent's confidence in $X$ on the supposition that $Y$ is true. Finally,

(IC) For any statements $H, E$ and $b, E$ (incrementally) confirms $H$ relative to $b$ if and only if $\operatorname{Pr}(H \mid E \& b)>\operatorname{Pr}(H \mid b)$.

Notice that (IC), likewise (RHD1), does not require $E$ to be verified to confirm $H$. Yet, when (IC) is used to ascertain whether $H$ is confirmed by evidence that one actually has, it is natural, and indeed customary, to interpret $E$ as representing such actual evidence.

It is possible to "translate" (TD) into the following probabilistic rule: 
(BTD) For any statements $H, E$ and $b$, if $\operatorname{Pr}(H \mid b) \in(0,1)$ and $H \& b$ entails $E$, for any arbitrary statement $E^{*}$ such that $\operatorname{Pr}\left(E \vee E^{*} \mid b\right) \in(0,1), E \vee E^{*}$ confirms $H$ relative to $b$.

(BTD) is a logical consequence of (IC) in conjunction with the theorem of the probability calculus that if $X \& Z$ entails $Y$, and $\operatorname{Pr}(X \mid Z)$ and $\operatorname{Pr}(Y \mid Z) \in(0,1)$, then $\operatorname{Pr}(X \& Y \mid Z)>\operatorname{Pr}(X \mid Z)$.

When (BTD) is used to ascertain whether $H$ is confirmed by a piece of evidence that one actually has, represented by $E \vee E^{*}$, (BTD) allows this disjunction to confirm $H$ even if it is intuitive that $E \vee E^{*}$ cannot do so. If we re-formulate the analysis of the tacking by disjunction paradox in terms of actual evidence, this happens in cases (II-irr), (II-inc) and (III-inc), assuming also that in these cases $(H \mid b) \in(0,1)$ and $\operatorname{Pr}\left(E \vee E^{*} \mid b\right) \in(0,1)$. Bayesian methodology thus apparently faces difficulties strictly analogous to those affecting Kuipers' version of Hypotheticodeductivism. The Bayesian, unlike Kuipers' hypothetico-deductivist, is not however committed to claiming that any contingent but idiotic statement is confirmable. For she can reasonably equate the prior probability of any such statement to zero. The important question is whether Bayesian methodology offers any way to handle the other difficulties. I will now show that the answer is positive.

The Bayesian solution of these difficulties crucially depends on the application of the Total Evidence Condition. This is an ideal constraint for the analysis of confirmation process, initially proposed by Carnap and Hempel, requiring that a hypothesis' confirmation be estimated on the grounds of all evidence available in a given time. My claim is that the Bayesian can elude the problems raised by (BTD) if she accepts the Total Evidence Condition as a constraint for the application of the rule of incremental confirmation (IC) to actual cases of confirmation in science. The reason is that, though (BTD) allows $E \vee E^{*}$ taken as a single item to confirm $H$ given $b$ when it 
is intuitively impossible, whenever disjunctions like $E \vee E^{*}$ are parts of total evidence, the latter confirms $H$ given $b$ in full accordance with our insight.

The Bayesian may therefore avoid the tacking by disjunction problem by employing a three-step strategy: she should first argue that the Total Evidence Condition is a necessary constraint for the application of (IC) to actual scientific cases. Then, she should show that this constraint has the effect of making total evidence including tacked disjunctions confirm or not confirm theories and hypotheses in accordance with our intuitions. From these two premises, the Bayesian will conclude that (BTD) yields no significant difficulty for confirmation theory.

Let us evaluate the first step of this strategy. Let $S$ be a scientist who decides to keep working on a hypothesis $H$, or to prefer $H$ to a rival $H^{*}$, on the grounds of the evidence available to her in a given time. If the evidence considered by $S$ were just a part of the total evidence available to her in that time, $S$ 's decisions could be argued not to be fully rational. For considering all evidence available might for instance show that $H$ is in fact at odds with experimental results or that $H^{*}$ is more supported by observations than $H$. Hence, $S$ 's decisions had better be based on all evidence she has. Since the confirmation of hypotheses is primarily meant to ground scientists' decisions, the Total Evidence Condition appears to be an essential constraint for confirmation in science. Accordingly, insofar as (IC) applies to actual scientific cases, it should be constrained by The Total Evidence Condition. $^{12}$

Let us now turn to the second step of the Bayesian strategy. Let $H$ be a hypothesis or theory such that $\operatorname{Pr}(H \mid b) \in(0,1)$, where $b$ is background knowledge. Moreover, let $E$ be a logical consequence of $H \& b$ and let $E^{*}$ be any statement such that $\operatorname{Pr}\left(E^{*} \mid b\right) \neq 0$ and $\operatorname{Pr}\left(E \vee E^{*} \mid b\right) \in(0$, $1)$.

If the total evidence available to a scientist $S$ includes the disjunction $E \vee E^{*}$, it will also include, in certain cases, $E$ or $E^{*}$. In particular, total evidence will include $E$ in case (I), $E^{*}$ in case (II), and both $E$ and $E^{*}$ in case (III). It is intuitive that, in cases (I), (II) and (III), $E \vee E^{*}$ has $n o$ 
weight in the confirmation of $H$ given total evidence and $b$. In the sense that, if $E \vee E^{*}$ were not included among the statements describing total evidence, $H$ 's confirmation would stand untouched. The reason is that, in these three cases, $E \vee E^{*}$ is entailed by $E$ or $E^{*}$, which constitute evidence already available to $S$. Thus $E \vee E^{*}$ can add no further support for $H$ than the support already conveyed by $E$ or $E^{*}$. Quite the opposite, our insight says that, in case (IV), $E \vee E^{*}$ can have a weight in the confirmation of $H$ given total evidence and $b$. For, now, $E \vee E^{*}$ is entailed by no other evidence available to $S$. All these claims perfectly comply with simple consequences of the probability calculus.

The total evidence available to $S$ can be equated to a finite conjunction of statements $X \& E_{1} \&$ $E_{2} \& \ldots \& E_{\mathrm{n}}$, where $E_{1}, E_{2}, \ldots, E_{\mathrm{n}}$ are the statements which we want to focus on and $X$ stands for the remaining part of total evidence. Accordingly, in case (I), (II) and (III), the confirmation of $H$ given total evidence depends on, respectively, the following statements:

(1) $\operatorname{Pr}\left(H \mid X \&\left(E \vee E^{*}\right) \& E \& b\right)$;

(2) $\operatorname{Pr}\left(H \mid X \&\left(E \vee E^{*}\right) \& E^{*} \& b\right)$;

(3) $\operatorname{Pr}\left(H \mid X \&\left(E \vee E^{*}\right) \& E \& E^{*} \& b\right)$.

Since both $E$ and $E^{*}$ entail $E \vee E^{*},(1),(2)$ and (3) are respectively equivalent to:

(1.1) $\operatorname{Pr}(H \mid X \& E \& b)$;

(2.1) $\operatorname{Pr}\left(H \mid X \& E^{*} \& b\right)$;

(3.1) $\operatorname{Pr}\left(H \mid X \& E \& E^{*} \& b\right)$.

This clearly shows that, in cases (I), (II) and (III), $E \vee E^{*}$ is irrelevant for the confirmation of $H$ given total evidence and $b$. This fulfils our expectations. ${ }^{13}$ 
Let us now consider case (IV). Total evidence includes $E \vee E^{*}$ but neither $E$ nor $E^{*}$. Accordingly, the confirmation of $H$ given total evidence and $b$ will depend on the following statement:

(4) $\operatorname{Pr}\left(H \mid X \&\left(E \vee E^{*}\right) \& b\right)$.

Now there is no immediate way to reduce (4) to:

(4.1) $\operatorname{Pr}(H \mid X \& b)$.

Thus, in case (IV), $E \vee E^{*}$ can have a weight in the confirmation of $H$ given total evidence and $b$. This also fulfils our expectations.

This analysis shows that, from the Bayesian perspective, total evidence including tacked disjunctions confirms or does not confirm hypotheses in accordance with our intuitions. Since the Bayesian methodologist appears committed to the Total Evidence Condition, she is allowed to claim that (BTD) and the tacking by disjunction paradox yield no significant difficulty for her notion of confirmation.

\section{Conclusion: the hypothetico-deductivists cannot appeal to the Total Evidence Condition}

I have shown that, though the most recent hypothetico-deductivist attempts to settle the tacking by disjunction problem are objectionable, the Bayesian who endorses the Total Evidence Condition can settle it without difficulty. A question naturally arises at this point as to whether versions of Hypothetico-deductivism would also successfully avoid the tacking by disjunction problem if they were supplemented with the Total Evidence Condition. More exactly, the question is whether the counterintuitive consequences that follow from estimating confirmation on individual tacked 
disjunctions can be eliminated - by analogy with the Bayesian strategy - by requiring that hypothetico-deductive confirmation be normally estimated on the grounds of total evidence. Before concluding this paper, I wish to address this question. It is my conviction that the answer is negative.

To begin with, both (RHD) and Kuipers' Hypothetico-deductivism require for the confirmation of $H$ by $E$ given $b$ that $E$ be logically deducible from $H \& b$. Moreover, (RHD1) requires for the confirmation of a natural axiom $H$ of a theory $T$ by evidence $E$ given $b$ that $E$ be logically deducible from $T \& b$. Notice however that total evidence $E$ available in a given time will in general consist of a conjunction of heterogeneous statements that is not logically deducible from any theory or hypothesis (unless such a "theory" or "hypothesis" is $E$ itself). Consequently, for any $H$ and $b, E$ will normally not confirm $H$ given $b$. As this conclusion is strongly implausible, the strategy that appeals to the Total Evidence Condition appears unavailable to any of the versions of Hypotheticodeductivism considered above.

To elude this difficulty, hypothetico-deductivists might think of using some close surrogate to the Total Evidence Condition. For example, one might require that the confirmation of $H$ given $b$ be estimated on strongest consequence of the total evidence that proves hypothetico-deductively relevant for $H$ given $b$ (i.e. that deductively confirms or that falsifies $H$ given $b$ ). This entails that, if total evidence $F$ is consistent with $H \& b$, the confirmation of $H$ given $b$ should be estimated on the grounds of the strongest consequence of $F$ that confirms $H$ given $b$. Accepting this requirement would, however, yield results that do not comply with our insight into confirmation. Consider, for instance, the case in which $H$ is a new theory not yet empirically tested and total evidence $F$ is consistent with $H \& b$. Suppose also that $F$ is intuitively irrelevant to $H$ given $b . H \vee F$ is the strongest consequence of $F$ that deductively confirms $H$ given $b$ on (RHD) and on Kuipers' Hypothetico-deductivism. Therefore, on both formulations, $H$ proves confirmed by evidence given $b$, which is clearly unacceptable. 
In addition, this surrogate to the Total Evidence Condition would not resolve difficulties affecting Gemes' (RHD1). Consider, for instance, the case in which $H$ is a natural axiom of some theory, and $H \& b$ entails $E \vee E^{*}$, where $E$ appears positively relevant to $H$ given $b$ and $E^{*}$ is inconsistent with $H \& b$. Suppose also that total evidence $F$ appears positively relevant to $H$ given $b$ just because $F$ entails $E \vee E^{*}$ but neither disjunct. As indicated in Section 4, (RHD1) allows disjunctions like $E \vee E^{*}$ to confirm $H$ given $b$ in no case at all. The application of the constraint at stake would not help, as it would be unable to make $H$ confirmed by evidence given $b$ on (RHD1).

Gemes (1998: 17) has made an alternative proposal: he suggests describing total evidence as the conjunction of two statements $X$ and $E$, where $X$ is irrelevant to $H$ and $E$ is relevant to $H$, and to include $X$ in background knowledge. Here, plausibly, a statement $S$ is considered irrelevant to $H$ if and only if, if $S$ is taken apart from total evidence, $S$ neither deductively confirms nor falsifies $H$ given background knowledge (before the inclusion of $X$ ). On the other hand, $S$ is relevant to $H$ if and only if, if $S$ is considered apart from total evidence, $S$ deductively confirms or falsifies $H$ given background knowledge (before the inclusion of $X$ ).

Following Gemes, forms of relative Hypothetico-deductivism could be supplemented by what we could name the Total Evidence Condition*; namely, the constraint to the effect that:

The confirmation of a hypothesis $H$ must normally be estimated on the grounds of the relevant part $E$ of total evidence $E \& X$, where the irrelevant part $X$ of total evidence must be included in background knowledge.

The appeal to the Total Evidence Condition* might initially appear successful, at least when (RHD) and Kuipers' Hypothetico-deductivism are accepted. Consider again the theory $H$ not yet empirically tested and total evidence $F$ irrelevant to $H$ given $b$. One could argue that since $F$ is irrelevant to $H$ given $b, F$ coincides with the part $X$ of total evidence to be included in background 
knowledge. Let us name $b^{*}$ background knowledge after the inclusion of $F$. There is now no left part $E$ of total evidence that confirms $H$ given $b^{*}$. This fulfils our expectations.

The Total Evidence Condition* is however problematic because debars evidential statements not consisting in tacked disjunctions from confirming hypotheses in situations that would count as confirmation cases on both (RHD), (RHD1) and Kuipers' Hypothetico-deductivism. Consider, for instance, the case in which the total evidence for the hypothesis $(x)(F x \rightarrow Q x)$, with background $b$ tautologous, is $(F a \rightarrow Q a) \& Q a .(x)(F x \rightarrow Q x) \& b$, but not $b$ alone, entails $F a \rightarrow Q a$, and both $(x)(F x \rightarrow Q x) \& b$ and $F a \rightarrow Q a$ are contingent. Consequently, on (RHD) and Kuipers' Hypothetico-deductivism, $F a \rightarrow Q a$ confirms $(x)(F x \rightarrow Q x)$ given $b$. In addition, all relevant models of $F a \rightarrow Q a$ can be extended to relevant models of $(x)(F x \rightarrow Q x) \& b, F a \rightarrow Q a$ is thus part of the content of $(x)(F x \rightarrow Q x) \& b$. Furthermore, $\{(x)(F x \rightarrow Q x)\}$ is a natural axiomatization of the deductive closure of $\{(x)(F x \rightarrow Q x)\}$ and there is no alternative natural axiomatization $N$ such that, for some subset $s$ of the axioms of $N, F a \rightarrow Q a$ is a content part of $s \& b$ and $(x)(F x \rightarrow Q x)$ is not a content part of $s \& b$. Thus, $F a \rightarrow Q a$ confirms $(x)(F x \rightarrow Q x)$ relative to $b$ also on (RHD1). Finally, on both (RHD), (RH1) and Kuipers' Hypothetico-deductivism, $Q a$ proves irrelevant to $(x)(F x \rightarrow$ $Q x)$ given $b$, as $(x)(F x \rightarrow Q x) \& b$ neither entails $Q a$ nor is not inconsistent with it.

The conclusion seems to be that $F a \rightarrow Q a$ is a suitable candidate for the relevant $E$, and $Q a$ is a suitable candidate for the irrelevant $X$. $Q a$ should thus be included in background knowledge $b^{*}$. Yet, in that case, $\mathrm{Fa} \rightarrow Q a$ would no longer confirm $(x)(F x \rightarrow Q x)$ given $b^{*}$ on (RHD) and Kuipers' Hypothetico-deductivism, as $b^{*}$ alone entails $F a \rightarrow Q a$. Moreover, $F a \rightarrow Q a$ would no longer confirm $(x)(F x \rightarrow Q x)$ given $b^{*}$ on (RHD1) because $F a \rightarrow Q a$ is not a content part of $(x)(F x$ $\rightarrow Q x) \& b^{*}$. For the relevant models for $F a \rightarrow Q a$ that assign $F$ to $Q a$ cannot be extended to a relevant model of $(x)(F x \rightarrow Q x) \& Q a .^{14}$

A further difficulty is exposed by Gemes himself. The Total Evidence Condition* requires us to partition all information included in total evidence into exactly two parts corresponding to the 
relevant part $E$ and the irrelevant part $X$. However, this does not appear to be always possible. Consider the case in which the total evidence for the hypothesis $(x) F x$ is $F a \&(F a \rightarrow G b)$, and suppose, again, that background knowledge $b$ is tautologous. Although $F a$ is relevant to $(x) F x$, and $F a \rightarrow G b$ is irrelevant to it, $F a$ and $F a \rightarrow G b$ are not suitable candidates for, respectively, the relevant part $E$ of total evidence and the irrelevant part $X$ of total evidence. (Cf. ibid.). For, if $\mathrm{Fa}$ and $\mathrm{Fa} \rightarrow \mathrm{Gb}$ are teased apart, some of the information included in total evidence goes astray, since $\mathrm{Fa}$ $\&(F a \rightarrow G b)$ entails $G b$ but neither $F a$ nor $F a \rightarrow G b$ alone does.

To settle this difficulty, Gemes cautiously suggests imposing a canonical way to describe total evidence that requires that 'both $E$ and $[\ldots X]$ be content parts of our total evidence' (ibid.), where 'content parts' is to be intended in Gemes' specific sense. ${ }^{15}$ Notice in fact that $F a \rightarrow G b$ is not a content part of $F a \&(F a \rightarrow G b)$. Plausibly, Gemes believes that, once this requirement is accepted, total evidence $F a \&(F a \rightarrow G b)$ can be split up into the relevant $F a$ and the irrelevant $G b$. For $F a \&$ $G b$ is logically equivalent to $F a \&(F a \rightarrow G b)$, and $F a$ and $G b$ are both content parts of it. In this case, there is no apparent loss of information.

Unfortunately, there are cases of loss of information that cannot be prevented in this way. Consider again the hypothesis $(x)(F x \rightarrow Q x)$ with background knowledge $b$ tautologous. Suppose that total evidence amounts to $(F a \rightarrow Q a) \&(Q a \rightarrow R a)$. Both $F a \rightarrow Q a$ and $Q a \rightarrow R a$ are content parts of total evidence. Moreover, while $F a \rightarrow Q a$ is relevant to $(x)(F x \rightarrow Q x)$ given $b, Q a \rightarrow R a$ is not. $F a \rightarrow Q a$ and $Q a \rightarrow R a$ are thus candidates for, respectively, the relevant $E$ and the irrelevant $X$. Notice however that, if $\mathrm{Fa} \rightarrow Q a$ and $Q a \rightarrow R a$ are teased apart, some of the information included in the total evidence gets irremediably lost. For $(F a \rightarrow Q a) \&(Q a \rightarrow R a)$ entails $F a \rightarrow R a$ but neither $\mathrm{Fa} \rightarrow Q a$ nor $Q a \rightarrow R a$ alone does.

Another problem of Gemes' requirement is that it prevents evidential statements that are not tacked disjunctions from confirming hypotheses in situations that count as cases of confirmation on both (RHD), (RHD1) and Kuipers' Hypothetico-deductivism. Consider for instance the situation in 
which the total evidence available for the hypothesis $(x)(F x \rightarrow(Q x \& R x))$, with background $b$ tautologous, is $(F a \rightarrow(Q a \& R a)) \& Q a . F a \rightarrow(Q a \& R a)$ confirms $(x)(F x \rightarrow(Q x \& R x))$ given $b$ on (RHD), (RHD1) and Kuipers' Hypothetico-deductivism. However, $F a \rightarrow(Q a \& R a)$ is not a content part of total evidence $(F a \rightarrow(Q a \& R a)) \& Q a$. As the relevant models of $F a \rightarrow(Q a \& R a)$ that assign $F$ to $Q a$ cannot be extended to relevant models of $(F a \rightarrow(Q a \& R a)) \& Q a$. Consequently, $F a \rightarrow(Q a \& R a)$ is not a candidate for $E$, and it cannot confirm $(x)(F x \rightarrow(Q x \&$ $R x)$ ) given $b^{*}$ if Gemes' requirement is accepted.

As I see no immediate solutions for these difficulties, the strategy that appeals to the Total Evidence Condition to resolve the tacking by disjunction problem appears to me unavailable to the hypothetico-deductivist.

\section{Acknowledgments}

I wish to thank Claus Beisbart, Robert Bishop, Luc Bovens, Franz Dietrich, Ludwig Fahrbach, Ken Gemes, Rolf Haenni, Hykel Hoshi, Franz Huber, Iain Martel, Phillip Meadows, David Papineau, Tommaso Piazza, and two referees of this Journal for valuable comments and suggestions upon early versions of this paper. My research was supported by the Center for Junior Research Fellows (ZWN) of the University of Konstanz and, in form of a Sofja Kovalevskaja Award, by the Alexander von Humboldt Foundation, the Federal Ministry of Education and Research and the Program for the Investment in the Future (ZIP) of the German Government.

\section{Notes}

${ }^{1}$ A confirmation rule strictly analogous to (TD) logically follows from (HD). The tacking by disjunction problem should however be formulated to apply to (RHD) and not to (HD). The reason is that the analysis of this problem and the appreciation of why it is a problem presuppose examining information usually stored in background knowledge. Without any reference to 
background information, it will in many cases be unclear whether a disjunction like $E \vee E^{*}$ intuitively confirms or does not confirm $H$. This will become apparent in the next section.

${ }^{2}$ This analysis assumes that whether $E \vee E^{*}$ can provide some amount of confirmation, in an intuitive sense, to $H$ given $b$ depends on whether or not $E \vee E^{*}$ is known or verified. Insofar as the relevant notion of confirmation is the intuitive or pre-theoretical one, it seems in fact true that a statement counts as confirming evidence only if is verified. For instance, we pre-theoretically assume that some of our empirical beliefs, but not all our empirical beliefs, are supported or confirmed by evidence (where an empirical belief is a confirmable one). This assumption makes sense if the notion of confirmation appealed to entails that a statement counts as confirming evidence only if is verified. If the notion of confirmation did not entail this, the assumption at stake would be incoherent. For we should conclude that any empirical belief is confirmed by default namely, by its empirical consequences, whether they are verified or not. (Focusing on refuting or falsifying evidence, we should conclude that any empirical belief is falsified by default). Furthermore, we pre-theoretically assume that a given belief, for instance, has been confirmed, or will be confirmed, or has not yet been confirmed, and so on and so forth. The meaningful use of these tensed expressions, in these phrases, appears to presuppose, again, that evidence counts as confirming evidence only if known. For example, one will normally interpret the phrase 'this belief has not yet been confirmed' in the sense that no evidence relevant to that belief has been attained (or known) so far. The same result obtains if we focus on expressions of common use in science, such as 'this is a refuted hypothesis' or 'this is a well confirmed theory'. These features of the pretheoretic notion of confirmation may cast doubts on the intuitive adequacy of formal accounts of confirmation that, like certain types of Hypothetico-Deductivism and standard Bayesian confirmation theory, do not assume that confirming evidence need to be known evidence. To argue that my analysis of cases (I)-(IV) rests on a counterintuitive notion of confirmation would probably mean to put the cart before the horse. Quite the opposite, I believe that this analysis shows that the 
conceptual framework underlying certain formal accounts of confirmation is too rudimentary to illuminate the nature of the confirmation paradox scrutinised in this paper.

${ }^{3}$ For instance, I assume that $\mathrm{Mr} X$ 's is not blind. Such and similar assumptions can be included in background knowledge.

${ }^{4}$ See Hempel (1966: 64). On this principle, see also Grimes (1990).

${ }^{5}$ Namely the paradox that if $H$ is confirmed relative to $b$ by the consequence $E$ of $H \& b$, for any arbitrary statement $H^{*}$ such that $H \& H^{*} \& b$ is contingent, $E$ also confirms $H \& H^{*}$ relative to $b$. See Gemes (1998: 1 and 10).

${ }^{6}$ Let us prove that if $X$ confirms $H$ given $b$ on (RHD1), and $H \& b$ entails $X, X$ is a content part of $H$ $\& b$. (This entails the statement at stake). If $X$ confirms a natural axiom $H$ of a theory $T$ given $b$ on (RHD1), then, for any natural axiomatisation $N(T)$ of $T$ and any subset $s$ of $N(T)$ such that $X$ is a content part of $s \& b, H$ is also a content part of $s \& b$. Therefore, for any such $s, s \& b$ entails $H \&$ $b$. As, by assumption, $H \& b$ entails $X$ (and both $H \& b$ and $X$ are contingent) $X$ may not be a content part of $H \& b$ only if there is a relevant model of $X$ that cannot be extended to a relevant model of $H \& b$. But this is impossible, as, for any $s, X$ is a content part of $s \& b$, which entails $H \&$ $b$.

${ }^{7}$ For example, where $F a$ confirms $(x) F x$, (RHD1) does not allow confirmation to "spill over" to the other parts of $(x) F x$ that extend beyond $F a-$ e.g., $F b$ and $F c$. Notice that $F b$ and $F c$ do not entail $F a$. Cf. Gemes (1998: 11).

${ }^{8}$ For further problems affecting (RHD1), see S. J. Park (2004).

${ }^{9}$ Although Kuipers is not explicit on this, he surely conceives of $E$ as a non-tautologous statement.

${ }^{10}$ The other three cases are, for Kuipers, the following: $H$ entails $\neg E$ (falsification), $\neg H$ entails $E$ (deductive disconfirmation), $\neg H$ entails $\neg E$ (verification).

11 This would seem to vindicate Glymour's suggestion that it is better for a definition of confirmation to be too exclusive than too inclusive. Cf. Gemes (1998: 13). 
${ }^{12}$ Earman (1992: 34) and Howson and Urbach (1993: 258) emphasize that the Total Evidence Condition is a necessary constraint for the application of the rule of strict conditionalisation. Let $\operatorname{Pr}$ $(X \mid Y)$ express the degree of confidence that a rational agent has in $X$ when she supposes that $Y$ is true. It seems correct to believe that after learning $Y$, the rational agent's degree of confidence in $X$ becomes equal to $\operatorname{Pr}(X \mid Y)$ if $Y$ is all evidence she has. Notice that, from this, it does not seem to follow that the Total Evidence Condition is also a constraint for the application of the rule of incremental confirmation.

${ }^{13}$ It might be thought that, if $E^{*}$ is irrelevant to $H$ 's confirmation given $b,(2.1)$ and (3.1) should in turn prove equivalent to, respectively:

(2.2) $\operatorname{Pr}(H \mid X \& b)$;

(3.2) $\operatorname{Pr}(H \mid X \& E \& b)$.

Yet this does not follow from the standard notion of (relative) probabilistic independence I rely on and that I find acceptable. In fact, $\operatorname{Pr}\left(H \mid E^{*} \& b\right)=\operatorname{Pr}(H \mid b)$ does not entail that, for any statement $X, \operatorname{Pr}\left(H \mid E^{*} \& X \& b\right)=\operatorname{Pr}(H \mid X \& b)$. It might however be thought that there is a notion of irrelevance that allows (2.2) and (3.2) to follow from (2.1) and (3.1); precisely: $E^{*}$ is irrelevant for $H^{\prime}$ 's confirmation given $b$ if and only if (i) $\operatorname{Pr}\left(H \mid E^{*} \& b\right)=\operatorname{Pr}(H \mid b)$ and (ii), for every statement $X$, $\operatorname{Pr}\left(H \mid E^{*} \& X \& b\right)=\operatorname{Pr}(H \mid X \& b)$. As Gemes has indicated (personal communication), the problem is that if (i) is satisfied, (ii) cannot be satisfied with $\operatorname{Pr}(H \mid b) \in(0,1)$. For, when these conditions hold and $X$ coincides with $E^{*} \rightarrow H, \operatorname{Pr}\left(H \mid E^{*} \&\left(E^{*} \rightarrow H\right) \& b\right) \neq \operatorname{Pr}\left(H \mid\left(E^{*} \rightarrow H\right) \& b\right)$.

${ }^{14}$ One referee of this Journal has suggested that saying that $F a \rightarrow Q a$ confirms $(x)(F x \rightarrow Q x)$ given $b$ tautologous looks like an appeal to the restricted Nicod Condition, which is known to be subject to counterexamples (see, for instance, Howson and Urbach 1993: 126-130). This is may be correct. Notice however that non-tautologous $b$ could plausibly be specified in a way to make it intuitive 
that $F a \rightarrow Q a$ confirms $(x)(F x \rightarrow Q x)$ relative to $b$. Thus, though there may be cases in which it is counterintuitive that $F a \rightarrow Q a$ confirms $(x)(F x \rightarrow Q x)$ given $b$, there may also be cases in which this is not counterintuitive. Gemes' proposal, if implemented, would have the effect to rule out many of the good and the bad cases at the same time. Whether there is a sense in which this would be beneficial to the hypothetico-deductivist models of confirmation considered in this paper is a hard question that should be thoroughly investigated. What it is certain is that Gemes' proposal is incoherent with his own formulation of relative Hypothetico-deductivism and the others considered in this paper.

${ }^{15}$ Gemes hastens to say that 'These are matters that deserve much greater elaboration within the context of a total theory of confirmation'. Gemes (1998: 17).

\section{References}

Earman, J. 1992, Bayes or Bust? A Critical Examination of Bayesian Confirmation Theory. MIT Press, Cambridge, Mass.

Gemes, K. 1994. 'A New Theory of Content'. Journal of Philosophical Logic 23: 596-620.

Gemes, K. 1997. ‘A New Theory of Content II: Model Theory and Some Alternatives'. Journal of Philosophical Logic 26: 449-476.

Gemes, K. 1998. 'Hypothetico-Deductivism: the Current State of Play; the Criterion of Empirical Significance: Endgame'. Erkenntnis 49: 1-20.

Grimes, T. R. 1990. 'Truth, Content, and the Hypothetico-Deductive Method'. Philosophy of Science 57: 514-522.

Hempel, C. 1966. Philosophy of Natural Science. Prentice Hall, Englewood Cliffs.

Howson C. and Urbach P. 1993. Scientific Reasoning: The Bayesian Approach. Open Court.

Kuipers, T. A. F. 2000. From Instrumentalism to Constructive Realism. Kluwer, Dordrecht.

Park. S. J. 2004. 'Hypothetico-Deductivism is Still Hopeless'. Erkenntnis 60: 229-234. 
Schurz, G. 1991. 'Relevant Deduction'. Erkenntnis 35: 391-437.

Schurz, G. 1994. 'Relevant Deduction and Hypothetico-Deductivism: A Reply to Gemes'. Erkenntnis 41: 183-188. 